\title{
Prevalencia de factores de riesgo cardiovascular en escolares de un área rural y de una urbana en Colombia
}

\author{
Germán Briceño, Jaime Céspedes, Martha Leal, Sarha Vargas \\ Fundación Cardioinfantil - Instituto de Cardiología, Bogotá, D.C., Colombia
}

Introducción. Los factores de riesgo cardiovascular tienen su origen en la infancia. En diversos estudios, se han registrado diferencias en la prevalencia de tales factores entre las áreas rurales y las urbanas, probablemente asociadas con los estilos de vida.

Objetivos. El presente estudio describe los factores de riesgo cardiovascular detectados en niños de una población rural y otra urbana en Colombia.

Materiales y métodos. Se hizo un estudio de corte transversal, entre marzo y junio del 2013, en escolares de un área urbana y de otra rural en Colombia. Se midieron el peso, la talla, la presión arterial, los triglicéridos, la glucemia y el colesterol total en ayunas y se hizo una encuesta sobre la dieta, la actividad física y el tabaquismo pasivo. Asimismo, se evaluaron las prevalencias de los factores de riesgo cardiovascular con un intervalo de confianza (IC) del $95 \%$.

Resultados. Se estudiaron 1.055 escolares, 833 en el área urbana y 222 en el área rural, cuyo promedio de edad fue de 6,71 años. Los factores de riesgo cardiovascular más prevalentes en la población rural y en la urbana fueron el sedentarismo (68,69 \% y 90,16\%, respectivamente), una dieta no saludable $(97,18 \%$ y $95,44 \%)$, el tabaquismo pasivo ( $11,16 \%$ y $14,52 \%)$, la obesidad $(0 \%$ y $5,64 \%$ ), la hipertensión arterial $(6,31 \%$ y $11,28 \%$ ), la diabetes ( $0 \%$ y $0 \%$ ), y la hipercolesterolemia (18,28 \% y $16,31 \%)$. El $99,15 \%$ de la población de estudio presentó, por lo menos, un factor de riesgo con un promedio de 3,14 (desviación estándar, $D E=1,12$ ) en el área urbana, y de 2,76 $(D E=1,1)$ en la rural. Los niños con exceso de peso presentaron mayor prevalencia de hipertensión arterial $(15,21$ $\%$ ) y de sedentarismo (90,69 \%), comparados con aquellos sin exceso de peso, 8,98 \% y 84,32 \%, respectivamente.

Conclusiones. Los resultados evidenciaron una alta prevalencia de factores de riesgo cardiovascular en niños, mayor en el área urbana. Se requieren estrategias de salud pública adaptadas a la población rural y a la urbana.

Palabras clave: enfermedades cardiovasculares; factores de riesgo; salud pública; niño; población rural; población urbana.

doi: https://doi.org/10.7705/biomedica.v38i4.4223

\section{Prevalence of cardiovascular risk factors in schoolchildren from a rural and urban area in Colombia}

Introduction: Cardiovascular risk factors (CVRF) have their origin in childhood. Several studies have shown differences in the prevalence of CVRFs between rural and urban areas, probably related to lifestyle behaviors.

Objective: To describe the CVRFs identified in children from a rural and urban population in Colombia. Materials and methods: A cross-sectional study was conducted between March and June 2013 in schoolchildren from an urban and a rural area in Colombia. Weight, height, blood pressure, triglycerides, fasting glucose, and total cholesterol were measured, and a survey covering nutrition, physical activity, and passive smoking was applied. The prevalence of CVRFs was calculated with a $95 \% \mathrm{Cl}$.

Results: A total of 1,055 schoolchildren (833 urban, 222 rural) participated; their mean age was 6.71 years. The prevalence of CVRFs in the rural and the urban study population, respectively, was $68.69 \% / 90.16 \%$ for sedentary lifestyle, $97.18 \% / 95.44 \%$ for unhealthy diet, $11.16 \% / 14.52 \%$ for passive smoking, $0 \% / 5.64 \%$ for obesity, $6.31 \% / 11.28 \%$ for hypertension, $0 \% / 0 \%$ for diabetes, and 
$18.28 \% / 16.31 \%$ for total cholesterol. A total of $99.15 \%$ of the study population had at least one CVRF, with an average of 3.14 for the urban area (SD:1.12), and of 2.76 for the rural one (SD: 1.1). Overweight children had a higher prevalence of hypertension (15.21\%; 95\% Cl:11.04\%-20.59\%) and sedentary lifestyle (90.69\%), compared to those without this risk factor (8.98\% and $84.32 \%$, respectively).

Conclusions: Our results showed a high prevalence of CVRFs in children, especially in the urban area. Public health strategies adapted to the rural and urban populations should be implemented.

Key words: Cardiovascular diseases; risk factors; public health; child; rural population; urban population.

doi: https://doi.org/10.7705/biomedica.v38i4.4223

La enfermedad cardiovascular es la primera causa de mortalidad a nivel mundial. Según un informe del Banco Mundial del 2007, el $56 \%$ de todas las muertes en países de ingresos bajos y medios se pueden atribuir a enfermedades no transmisibles (1), y para el 2020 , el $80 \%$ de todas las muertes podrá atribuirse a estas enfermedades (2). Los comportamientos nocivos para la salud iniciados desde la niñez, como el sedentarismo, los malos hábitos nutricionales y la obesidad, que persisten en la edad adulta, pueden ser factores de riesgo de enfermedad cardiovascular cuyas consecuencias en la economía y en la salud son bien conocidas (3). A menudo, la enfermedad se origina en la ateroesclerosis, la cual comienza en la infancia, es de progresión lenta y puede prevenirse (4).

El crecimiento acelerado de la carga de la enfermedad cardiovascular afecta de forma desproporcionada a los países de ingresos bajos y medios, pues aumenta las diferencias en salud dentro de los países y entre ellos $(5,6)$. La prevalencia de los factores de riesgo cardiovascular se ha incrementado en los últimos años en todo el mundo $(7)$ y en Colombia $(8,9)$ ha comenzado a afectar a la población pediátrica al igual que en otros países latinoamericanos, como Ecuador (10), Brasil (11), Chile (12), México (13) y el mismo Colombia (14), en donde en un estudio en población urbana se estableció una alta prevalencia de dieta no saludable $(89,0 \%)$, sedentarismo $(78,8 \%)$, tabaquismo pasivo $(19,5 \%)$, historia familiar de riesgo cardiovascular $(16,1 \%)$ y obesidad $(12,7$ $\%)$, sin diferencias significativas según el sexo.

En muchos países latinoamericanos, se vive un proceso creciente y acelerado de concentración de la población en las áreas urbanas. A nivel mundial, la población rural ha disminuido, pasando del

\footnotetext{
Correspondencia:

Germán Briceño, Carrera 13b №163-85, torre A, piso 3, Bogotá, D.C., Colombia

Teléfono: (571) 667 2727, extensión 6300

dariobrice@gmail.com
}

$66,4 \%$ en 1960 al $45,7 \%$ en 2016 , en tanto que en Colombia (15) pasó de $54,9 \%$ a $23,3 \%$ (16). Las disparidades económicas en salud y en educación entre las zonas rurales y urbanas (17) pueden estar asociadas con los diferentes patrones de dietas y de actividad física, lo que, a su vez, se relaciona con el comportamiento de los factores de riesgo $(18,19)$.

Dado que, en gran medida, las enfermedades no transmisibles son prevenibles cuando se eliminan los factores de riesgo compartidos, como el tabaquismo, una dieta no saludable, la inactividad física y el consumo de alcohol, establecer la prevalencia de los factores de riesgo cardiovascular en la población infantil de una zona urbana y una rural en un país de ingresos bajos y medios, puede contribuir a la planificación de estrategias específicas de promoción de la salud cardiovascular. En el presente estudio se describen los factores de riesgo cardiovascular en niños de una población rural y de otra urbana en Colombia.

\section{Materiales y métodos \\ Diseño del estudio y participantes}

Entre marzo y junio de 2013 se desarrolló un estudio de corte transversal en dos poblaciones colombianas: una de nueve colegios públicos de un área rural del departamento de Boyacá, y otra de nueve colegios privados en la capital colombiana.

Se hizo un muestreo no probabilístico a conveniencia. Las mediciones corresponden a la línea basal de dos estudios clínicos actualmente en desarrollo liderados por los autores del presente estudio, uno en área rural y otro en área urbana, cuyo objetivo es evaluar el impacto de una intervención en los hábitos de alimentación de los niños. Se incluyeron niños entre 5 y 9 años de edad, de colegios mixtos y jornada académica única de siete o más horas al día, lo que facilita la oportunidad de hacer actividad física. Se excluyeron los niños de aquellos colegios en los que se hubieran implementado intervenciones de más de un mes de duración 
relacionadas con hábitos saludables (nutrición o actividad física) durante el año previo al inicio del estudio.

La población rural objetivo residía en la vereda de Sotepanelas (población, 8.067 habitantes; altitud, $3.240 \mathrm{msnm}$; temperatura promedio, 11,4 ${ }^{\circ} \mathrm{C}$ ), situada a $8,5 \mathrm{~km}$ de Tunja, capital del departamento de Boyacá, cuya participación en el producto interno bruto (PIB) del país es de 2,9\%, y en donde solo hay colegios públicos con jornada académica única.

La población urbana objetivo se ubicaba en Bogotá (población, 8'080.000 habitantes; altitud, $2.640 \mathrm{msnm}$; temperatura promedio, $14^{\circ} \mathrm{C}$ ), cuya participación en el PIB del país es de 25,7 \%, y en donde solo los colegios privados tienen jornada académica única.

Los participantes y sus acudientes firmaron el consentimiento informado. El diseño del estudio, la recolección de datos y su análisis, se llevaron a cabo bajo la supervisión y con la aprobación del comité de ética institucional.

\section{Mediciones del estudio}

La definición de factores de riesgo cardiovascular en niños y adolescentes utilizada en el estudio, se ajustó a las guías de salud cardiovascular y reducción de riesgo en niños y adolescentes del Expert Panel on Integrated Guidelines for Cardiovascular Health and Risk Reduction in Children and Adolescents (20) y las definicio nes de la American Heart Association (AHA) (21) (cuadro 1).

Antes de hacer las encuestas y tomar las muestras y medidas, el equipo del estudio recibió capacitación en la estandarización del uso de las encuestas, la técnica de recolección de la muestra sanguínea, y la toma de las medidas antropométricas en población rural y urbana.

Los hábitos de la dieta, de actividad física y el tabaquismo pasivo en la semana anterior se evaluaron mediante un cuestionario basado en la Global School-based Student Health Survey (GSHS) de los Centers for Disease Control and Prevention (CDC) de Estados Unidos (22). La encuesta fue respondida por los niños acompañados de sus padres. Las preguntas incluyeron la información sobre el consumo de frutas, verduras y comida chatarra en las comidas principales y entre comidas, así como sobre la actividad física y la práctica de actividades sedentarias. La toma de muestras sanguíneas en ayunas para el análisis de glucemia, triglicéridos y colesterol total estuvo a cargo de enfermeras bajo estándares de asepsia, y las muestras se procesaron en el laboratorio central de la Fundación Cardiolnfantil.

Para la evaluación del índice de masa corporal (IMC), se midió la estatura con un tallímetro manual (Hyssna Measuring Equipment $A B$, Suecia) y el peso con una báscula digital (Tanita Corporation ${ }^{\mathrm{TM}}$, Japón). Durante ambas mediciones, los niños vestían ropa ligera y estaban descalzos.

Todas las mediciones se hicieron tres veces con el valor más cercano a $0,1 \mathrm{~cm} / 0,1 \mathrm{~kg}$, y se registró el promedio en caso de diferencias. El IMC se calculó como el peso en kilogramos dividido por la altura en metros cuadrados.

Para la medición de la presión arterial, los menores debían haber estado en reposo por lo menos durante cinco minutos antes de la medición, y no haber consumido medicamentos ni alimentos estimulantes. Los niños estaban sentados con la espalda apoyada y los pies en contacto con el piso, el brazo derecho sobre un soporte y la fosa cubital colocada a nivel del corazón. La presión arterial sistémica se tomó tres veces con brazaletes apropiados para la edad localizados en un punto medio entre el acromion y el olécranon mediante técnicas estandarizadas (23). Los niveles de presión arterial diastólica entre 90 y $95 \mathrm{~mm} \mathrm{Hg}$ se consideraron indicativos de prehipertensión arterial sistémica.

El estado nutricional de los niños se calculó con base en el IMC por edad y sexos, según lo establecido por la Organización Mundial de la Salud (OMS): desnutrición aguda, IMC $\leq 2$ desviación estandar (DE); riesgo de malnutrición, entre $-2 \mathrm{DE}$ y $\leq 1 \mathrm{DE}$; eutrofia, entre $-1 \mathrm{DE}$ y $1 \mathrm{DE}$; sobrepeso, entre $>1$ DE y $2 \mathrm{DE}$, y obesidad, >2DE. El exceso de peso se definió como sobrepeso u obesidad (24).

\section{Análisis estadístico}

Se calcularon la prevalencia y los intervalos de confianza (IC) del $95 \%$ del estado nutricional y los factores de riesgo cardiovascular, incluidos el sedentarismo, la dieta no saludable, el tabaquismo pasivo, la hipertensión arterial sistémica, la obesidad, el colesterol total (>200 mg/dl) y la diabetes, considerando el sexo y el contexto rural o urbano de la población de estudio. Para las variables continuas, se estimaron la media 
Cuadro 1. Mediciones de los factores de riesgo cardiovascular

\begin{tabular}{|c|c|c|}
\hline & $\begin{array}{l}\text { Factor de riesgo cardiovascular, } \\
\text { definición de la } \mathrm{AHA}^{\mathrm{a}}\end{array}$ & $\begin{array}{l}\text { Factor de riesgo cardiovascular, } \\
\text { definición del estudio }\end{array}$ \\
\hline \multicolumn{3}{|l|}{ Comportamientos de salud } \\
\hline Estado de tabaquismo & Prueba $>30$ días & Tabaquismo pasivo ${ }^{\mathrm{b}}$ \\
\hline Índice de masa corporal (IMC) & IMC >percentil 95 & $\mathrm{IMC} \geq 2 \mathrm{DE}$ \\
\hline Nivel de actividad física & Ninguna & $\begin{array}{l}<60 \text { minutos/día de actividad moderada o } \\
\text { vigorosa } \leq 4 / \text { día a la semana y actividades } \\
\text { sedentarias }^{c}>2 \text { h/día }\end{array}$ \\
\hline \multirow[t]{6}{*}{ Dieta saludable } & $0-1$ componentes & Frutas y verduras $\leq 3 /$ día \\
\hline & Frutas y verduras: $\geq 4,5$ tazas/día & Comida chatarra >2/día \\
\hline & Pescado: $\geq 2$ porciones de 3,5 oz/semana & \\
\hline & $\begin{array}{l}\text { Granos enteros ricos en fibra: } \geq 3 \\
\text { porciones de } 1 \mathrm{oz} / \text { día }\end{array}$ & \\
\hline & Sodio: $<1.500$ mg/día & \\
\hline & $\begin{array}{l}\text { Bebidas azucaradas: } \leq 450 \mathrm{kcal}(36 \mathrm{oz}) / \\
\text { semana }\end{array}$ & \\
\hline \multicolumn{3}{|l|}{ Factores de salud } \\
\hline Colesterol total & $\geq 200 \mathrm{mg} / \mathrm{dl}$ & $\geq 200 \mathrm{mg} / \mathrm{dl}$ \\
\hline Presión arterial sistémica & >percentil 95 & >percentil 95 \\
\hline Glucemia en ayunas & $>126 \mathrm{mg} / \mathrm{dl}$ & $>126 \mathrm{mg} / \mathrm{dl}$ \\
\hline
\end{tabular}

a American Heart Association

b Tabaquismo pasivo: niños no fumadores que respiran el humo de fumadores activos

c Comportamientos sedentarios: ver televisión, jugar video juegos o juegos de computadores, o usar el computador para acciones diferentes a tareas 0 actividades del colegio

y la desviación estándar. No se hizo ningún tipo de depuración de las muestras para mantener un equilibrio. Se calcularon las razones de prevalencia $\left(\mathrm{IC}_{95 \%}\right)$ de los factores de riesgo cardiovascular ajustadas por edad y sexo mediante un análisis de regresión multivariado y utilizando modelos lineales generalizados. Los análisis se realizaron con Stata ${ }^{\mathrm{TM}} 13.1$.

\section{Resultados}

Se evaluaron 1.055 escolares, 833 de la población urbana y 222 de la rural, con un promedio de edad de 6,71 años, la mayor parte de ellos eutróficos (cuadro 2).

La prevalencia de factores de riesgo cardiovascular en la población de estudio, fue la siguiente: sedentarismo, 85,6 \% ( $\left.\mathrm{IC}_{95 \%} 83,30-87,63\right)$, dieta no saludable, $95,81 \% \quad\left(\mathrm{IC}_{95 \%} \quad 94,39-96,89\right)$; tabaquismo, 13,81\% (IC ${ }_{95 \%}$ 11,82-16,07); obesidad, $4,45 \% \quad\left(\mathrm{IC}_{95 \%} 3,37-5,87\right)$; hipertensión arterial sistémica, 10,23\% (IC $\left.{ }_{95 \%} 8,55-12,21\right)$; diabetes, $0,0 \%$, y colesterol total ( $\geq 200 \mathrm{mg} / \mathrm{dl}), 16,67 \%$ $\left(\mathrm{IC}_{95 \%}, 14,52-19,06\right)$. La información detallada y discriminada entre el área urbana y la rural, se presenta en el cuadro 3.
El 99,15\% de la población tenía, por lo menos, un factor de riesgo cardiovascular, con un promedio en la población urbana de $3,14(\mathrm{DE}=1,12)$, y de $2,76(D E=1,1)$ en la población rural.

\section{Comportamientos de salud}

Índice de masa corporal ( $n=1.055)$. El 20,66\% $\left(\mathrm{IC}_{95 \%} 18,33-23,21\right)$ de la población evaluada tenía exceso de peso (sobrepeso u obesidad) (cuadro 2); este fue mayor en el área urbana, con 24,49 $\%\left(\mathrm{IC}_{95 \%} 21,69-27,52\right)$, que en la rural, con $6,31 \%$ $\left(\mathrm{IC}_{95 \%} 3,79-10,31\right)$. Al ajustar por edad y sexo, la prevalencia de sobrepeso fue 2,98 veces mayor en la población urbana que en la rural (figura 1). La prevalencia de obesidad fue $10,62\left(\mathrm{IC}_{95 \%} 1,44\right.$ 78,01 ) veces mayor en la población urbana que en la rural $(p=0,020)$.

Los niños con exceso de peso tuvieron una mayor prevalencia de hipertensión arterial: 15,21\% ( $\mathrm{IC}_{95 \%}$ 11,04-20,59) y de sedentarismo: 90,69 \% ( IC $_{95 \%}$ 85,9-93,7), que aquellos sin exceso de peso: 8,98 \% $\left(\mathrm{IC}_{95 \%}, 7,23-11,11\right)$ y $84,32\left(\mathrm{IC}_{95 \%} 81,62-86,69\right)$, respectivamente.

Al ajustar por edad y sexo, la razón de prevalencia para la hipertensión arterial y el sedentarismo 
Cuadro 2. Características demográficas y del estado nutricional de la población urbana y rural

\begin{tabular}{|c|c|c|c|}
\hline & Población urbana & Población rural & Total \\
\hline \multirow[t]{2}{*}{ Característica } & $\mathrm{n}=833$ & $\mathrm{n}=222$ & $n=1055$ \\
\hline & Prevalencia \% $\left(\mathrm{IC}_{95 \%}\right)$ & Prevalencia \% $\left(\mathrm{IC}_{95 \%}\right)$ & Prevalencia \% $\left(\mathrm{IC}_{95 \%}\right)$ \\
\hline \multicolumn{4}{|l|}{ Sexo } \\
\hline \multirow[t]{2}{*}{ Masculino } & $(n=452)$ & $(n=119)$ & $(n=571)$ \\
\hline & $54,31(50,92-57,67)$ & $53,36(46,81-59,80)$ & $54,12(51,10-57,10)$ \\
\hline \multirow[t]{2}{*}{ Femenino } & $(n=381)$ & $(n=103)$ & $(n=484)$ \\
\hline & $45,68(42,33-49,08)$ & $46,63(40,20-53,19)$ & $45,88(42,90-48,90)$ \\
\hline Edad, media (DE) & $6,49(0,93)$ & $7,51(1,91)$ & $6,71(1,28)$ \\
\hline \multicolumn{4}{|l|}{ Estado nutricional $^{a}$} \\
\hline \multirow[t]{2}{*}{ Desnutrición aguda } & $(n=10)$ & $(n=5)$ & $(n=15)$ \\
\hline & $1,20(0,65-2,20)$ & $2,25(0,97-5,16)$ & $1,42(0,86-2,33)$ \\
\hline \multirow[t]{2}{*}{ Riesgo de desnutrición } & $(n=69)$ & $(n=20)$ & $(n=89)$ \\
\hline & $8,28(6,60-10,35)$ & $9,01(5,91-13,50)$ & $8,44(6,91-10,27)$ \\
\hline \multirow[t]{2}{*}{ Eutrofia } & $(n=550)$ & $(n=183)$ & $(n=733)$ \\
\hline & $66,03(62,74-69,16)$ & $82,43(76,89-86,87)$ & $69,48(66,63-72,18)$ \\
\hline \multirow[t]{2}{*}{ Sobrepeso } & $(n=157)$ & $(n=14)$ & $(n=171)$ \\
\hline & $18,85(16,34-21,64)$ & $6,31(3,79-10,31)$ & $16,21(14,11-18,55)$ \\
\hline \multirow[t]{2}{*}{ Obesidad } & $(n=47)$ & $(n=0)$ & $(n=47)$ \\
\hline & $5,64(4,27-8,02)$ & $0(0,00-1,17)$ & $4,45(3,37-5,87)$ \\
\hline
\end{tabular}

a Según la Organización Mundial de la Salud

en niños con exceso de peso fue de $1,53\left(\mathrm{IC}_{95 \%}\right.$ $1,14-2,04 ; p=0,004)$ y de $1,07\left(I_{95 \%} 1,02-1,13\right.$; $p=0,009)$, respectivamente.

Sedentarismo ( $n=1.008)$. El sedentarismo fue mayor en el área urbana que en la rural. Las niñas presentaron mayor prevalencia de sedentarismo que los niños, tanto en área urbana como rural (cuadro 3). Al ajustar por la edad y el sexo, la prevalencia fue 1,32 veces mayor en la población urbana que en la rural (figura 1).

Los niños sedentarios tuvieron más exceso de peso: 21,71 ( $\mathrm{IC}_{95 \%}$ 19,08-24,61) y de hipertensión arterial: $19,18\left(\mathrm{IC}_{95 \%} 16,68-21,96\right)$, que los no sedentarios: $13,29\left(\mathrm{IC}_{95 \%} 8,67-19,82\right)$ y $11,81\left(\mathrm{IC}_{95 \%}\right.$ $7,50-18,09)$, respectivamente. No se registraron diferencias significativas entre el colesterol de los niños sedentarios $(172,67 \mathrm{mg} / \mathrm{dl}$; $\mathrm{DE}=29,79)$ y el de los no sedentarios $(171,64 \mathrm{mg} / \mathrm{dl}$; DE=30,47), sin embargo, entre los niños con exceso de peso el nivel de colesterol fue mayor en los sedentarios $(173,21 \mathrm{mg} / \mathrm{dl} ; \mathrm{DE}=2,28)$ que en los no sedentarios (165,94 mg/dl; DE=7,33).

Dieta no saludable ( $n=1.003)$. El 95,81\% $\left(\mathrm{IC}_{95 \%}\right.$ 94,39-96,89) de la población de estudio consumía una dieta no saludable, con mayor participación de los niños del área rural (cuadros 1 y 3). Al ajustar por edad y sexo, se obtuvieron resultados similares (figura 1).

Tabaquismo $(n=1.014)$. El tabaquismo pasivo en niños fue mayor en el área urbana, sin diferencias significativas entre niños y niñas (cuadro 3). Al ajustar por edad y sexo, la prevalencia de tabaquismo pasivo fue mayor en la población urbana que en la rural, sin constituir una diferencia estadísticamente significativa (figura 1).

\section{Factores de salud}

Colesterol total y glucemia en ayunas ( $n=1.032)$. Los niveles de triglicéridos, colesterol total y glucemia de la población rural y en la urbana, se describen en el cuadro 4. Al ajustar por edad y sexo, la prevalencia de colesterol total (>200 mg/dl) fue mayor en la población rural que en la urbana, sin ser una diferencia estadísticamente significativa (figura 1). La concentración de triglicéridos en los niños con exceso de peso $(72,23 ; \mathrm{DE}=2,21)$ fue mayor que en los que no tenían tal exceso $(65,55$; $\mathrm{DE}=1,17)$.

La glucemia y el colesterol total no fueron diferentes en los niños con exceso de peso que en aquellos sin este; los niveles de glucemia fueron de 
Cuadro 3. Prevalencia de factores de riesgo cardiovascular en la población urbana y la rural por sexo

\begin{tabular}{|c|c|c|c|c|c|c|}
\hline \multirow{3}{*}{$\begin{array}{l}\text { Factor de riesgo } \\
\text { cardiovascular }\end{array}$} & \multicolumn{3}{|l|}{ Población urbana } & \multicolumn{3}{|l|}{ Población rural } \\
\hline & \multicolumn{3}{|c|}{ Prevalencia, \% (IC95\%) } & \multicolumn{3}{|c|}{ Prevalencia, \% (IC95\%) } \\
\hline & Niños & Niñas & Total & Niños & Niñas & Total \\
\hline $\mathrm{IMC}^{\mathrm{a}}$ & $\mathrm{n}=452$ & $\mathrm{n}=381$ & $\mathrm{n}=833$ & $\mathrm{n}=119$ & $n=103$ & $n=222$ \\
\hline Obesidad & $7,3(5,25-10,08)$ & $3,67(2,20-6,07)$ & $5,64(4,27-7,42)$ & $0(0,00-0,31)$ & $0(0,00-0,36)$ & $0(0,00-0,17)$ \\
\hline Sobrepeso & $17,92(14,66-21,72)$ & $19,95(16,24-24,25)$ & $18,85(16,34-21,64)$ & $6,72(3,45-12,71)$ & $5,83(2,77-12,13)$ & $6,31(3,79-10,31)$ \\
\hline \multirow[t]{2}{*}{ Sedentarismo } & $\mathrm{n}=425$ & $\mathrm{n}=368$ & $\mathrm{n}=793$ & $\mathrm{n}=113$ & $\mathrm{n}=101$ & $n=214$ \\
\hline & $88,94(85,60-91,58)$ & $91,58(88,29-94,00)$ & $90,16(87,89-92,05)$ & $61,95(52,74-70,37)$ & $76,24(67,07-83,48)$ & $68,69(62,19-74,53)$ \\
\hline \multirow[t]{2}{*}{ Dieta no saludable } & $\mathrm{n}=424$ & $n=366$ & $\mathrm{n}=790$ & $\mathrm{n}=113$ & $\mathrm{n}=100$ & $n=213$ \\
\hline & $96,7(94,53-98,02)$ & $93,99(91,07-96,00)$ & $95,44(92,55-95,76)$ & $98,23(93,78-99,51)$ & $96(90,16-98,43)$ & $97,18(93,99-98,70)$ \\
\hline Tabaquismo & $\mathrm{n}=428$ & $n=371$ & $\mathrm{n}=799$ & $\mathrm{n}=114$ & $\mathrm{n}=101$ & $\mathrm{n}=215$ \\
\hline Pasivo & $14,25(11,26-17,88)$ & $14,82(11,57-18,80)$ & $14,52(12,25-17,13)$ & $13,16(8,14-20,58)$ & $8,91(4,76-16,07)$ & $11,16(7,62-16,07)$ \\
\hline Presión arterial & $\mathrm{n}=452$ & $\mathrm{n}=381$ & $\mathrm{n}=833$ & $\mathrm{n}=119$ & $n=103$ & $\mathrm{n}=222$ \\
\hline PreHTA & $6,86(4,87-9,57)$ & $8,92(6,46-12,21)$ & $7,8(6,17-9,82)$ & $9,24(5,24-15,80)$ & $5,83(2,70-12,13)$ & $7,66(4,84-11,92)$ \\
\hline $\mathrm{HTA}^{\mathrm{b}}$ & $9,95(7,52-13,06)$ & $12,86(9,87-16,60)$ & $11,28(9,31-13,61)$ & $6,72(3,45-12,71)$ & $5,82(2,70-12,13)$ & $6,31(3,79-10,31)$ \\
\hline \multirow[t]{2}{*}{ Diabetes } & $\mathrm{n}=455$ & $n=389$ & $\mathrm{n}=844$ & $\mathrm{n}=101$ & $\mathrm{n}=85$ & $\mathrm{n}=186$ \\
\hline & $0(0,00-0,84)$ & $0(0,00-0,98)$ & $0(0,00-0,45)$ & $0(0,00-0,36)$ & $0(0,00-0,43)$ & $0(0,00-0,20)$ \\
\hline \multirow[t]{2}{*}{ Colesterol total $\geq 200 \mathrm{mg} / \mathrm{dl}$} & $\mathrm{n}=457$ & $\mathrm{n}=389$ & $\mathrm{n}=846$ & $\mathrm{n}=101$ & $\mathrm{n}=85$ & $\mathrm{n}=186$ \\
\hline & $14,22(11,32-17,72)$ & $18,77(15,20-22,94)$ & $16,31(13,98-18,95)$ & $16,83(10,78-25,31)$ & $20(12,88-29,72)$ & $18,28(13,39-14,46)$ \\
\hline
\end{tabular}

a IMC: índice de masa corporal

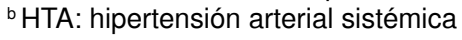

Cuadro 4. Biomarcadores e índice de masa corporal en la población urbana y en la rural

\begin{tabular}{|c|c|c|c|c|c|c|}
\hline \multirow{3}{*}{$\begin{array}{l}\text { Biomarcador por } \\
\text { grupo de edad }\end{array}$} & \multicolumn{3}{|c|}{ Población urbana } & \multicolumn{3}{|l|}{ Población rural } \\
\hline & Promedio (DE) & Promedio (DE) & Promedio (DE) & Promedio (DE) & Promedio (DE) & Promedio (DE) \\
\hline & Niños & Niñas & Total & Niños & Niñas & Total \\
\hline \multirow[t]{2}{*}{ Colesterol (mg/dl) } & $n=457$ & $n=389$ & $n=846$ & $n=101$ & $n=85$ & $n=186$ \\
\hline & $170,12(28,48)$ & $175,40(29,31)$ & $172,55(28,97)$ & $174,48(33,41)$ & $173,76(34,05)$ & $174,12(33,58)$ \\
\hline \multirow[t]{2}{*}{ Glucemia (mg/dl) } & $n=455$ & $n=389$ & $n=844$ & $n=101$ & $n=85$ & $n=186$ \\
\hline & $85,32(6,14)$ & $83,49(5,72)$ & $84,48(6,02)$ & $87,49(6,09)$ & $84,69(7,15)$ & $86,09(6,75)$ \\
\hline \multirow[t]{2}{*}{ Triglicéridos (mg/dl) } & $\mathrm{n}=457$ & $n=390$ & $n=847$ & $n=101$ & $\mathrm{n}=85$ & $n=186$ \\
\hline & $59,16(25,50)$ & $67,25(29,85)$ & $62,89(27,87)$ & $101,58(41,73)$ & $102,27(46,23)$ & $101,92(43,84)$ \\
\hline \multirow[t]{2}{*}{ IMCa (kg/m2) } & $n=452$ & $n=381$ & $n=833$ & $\mathrm{n}=119$ & $n=103$ & $n=222$ \\
\hline & $16,27(2,32)$ & $16,24(2,13)$ & $16,26(2,24)$ & $16,11(1,47)$ & $15,86(2,43)$ & $15,99(1,98)$ \\
\hline
\end{tabular}

a Índice de masa corporal

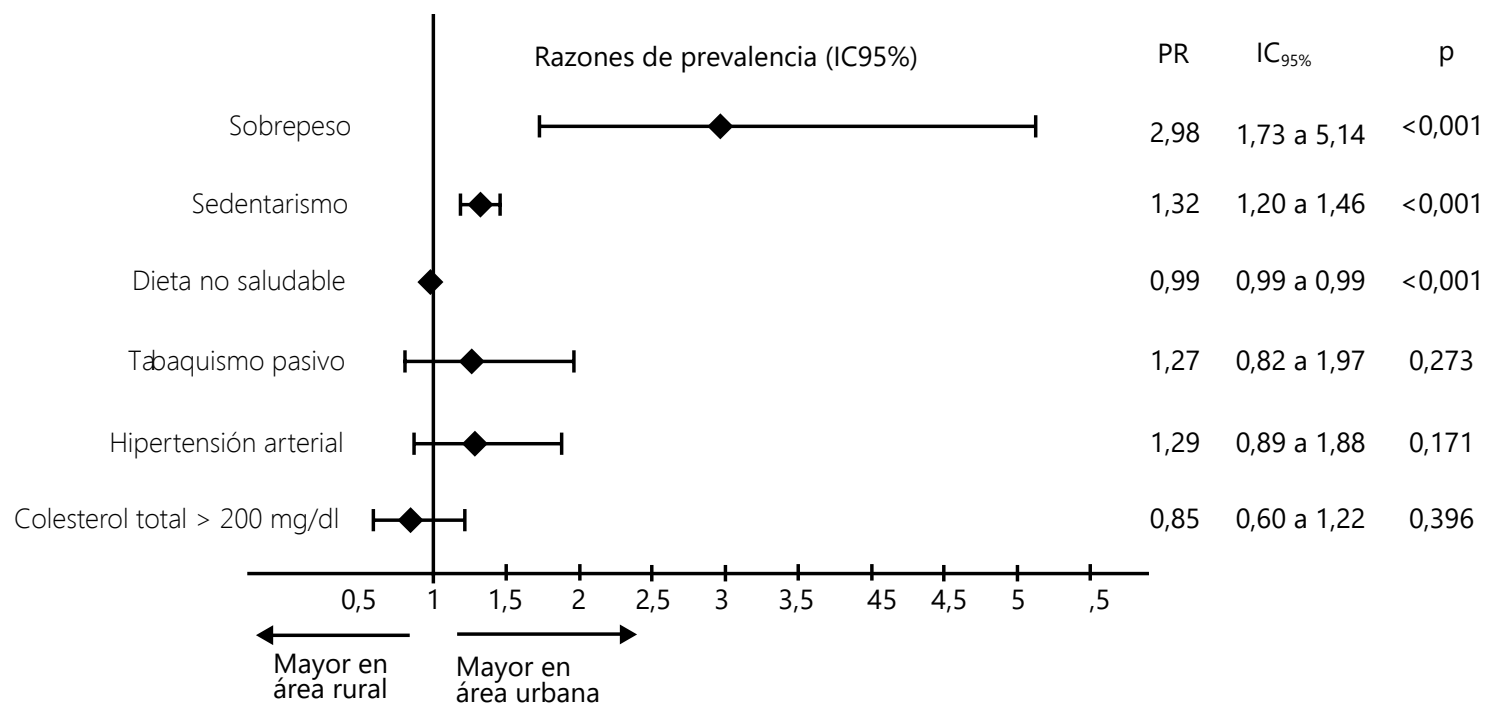

Figura 1. Razones de prevalencia de factores de riesgo cardiovascular en la población urbana y en la rural, ajustadas por edad y sexo 
$85,13 \mathrm{mg} / \mathrm{dl}(\mathrm{DE}=0,45)$ y de $84,45 \mathrm{mg} / \mathrm{dl}(\mathrm{DE}=0,23)$, respectivamente, y los de colesterol total de 172,83 $\mathrm{mg} / \mathrm{dl}(\mathrm{DE}=30,37)$ y de $172,71 \mathrm{mg} / \mathrm{dl}(\mathrm{DE}=29,56)$, respectivamente.

Presión arterial sistémica $(n=1055)$. La mayoría de los niños tenía una presión arterial sistémica normal. La prevalencia de hipertensión arterial fue mayor en el área urbana sin diferencias significativas entre niños y niñas (cuadro 3). Al hacer el ajuste por edad y sexo, se obtuvieron resultados similares (figura 1 ).

\section{Discusión}

En el presente estudio se encontró una alta prevalencia de factores de riesgo cardiovascular en escolares de una zona urbana y una rural en Colombia. Con excepción de la alta prevalencia de una dieta no saludable en la población rural, las prevalencias de sobrepeso, obesidad y sedentarismo fueron mayores en la población urbana (cuadros 3 y 4) (figura 1).

Estos hallazgos podrían utilizarse en la planeación de estrategias de promoción de la salud cardiovascular en escolares rurales y urbanos, dado que todos los factores de riesgo descritos son modificables.

En la población de estudio, la prevalencia de exceso de peso $(20,66 \%)$ fue menor que la reportada $(24,4 \%)$ en la presentación preliminar de la Encuesta Nacional de la Situación Nutricional en Colombia (ENSIN, 2015) (25) y menor que la reportada en Estados Unidos, de 34,2 \%(26). Asi mismo, la obesidad encontrada en niños fue de 5,95 $\%$ y de 2,89 \% en niñas, menor que la hallada en Estados Unidos, donde los niños presentaron una prevalencia de 19,6 \% y, las niñas, una de 16,6\% (27).

Aunque la magnitud del exceso de peso es mayor en Colombia, la población de estudio registró niveles inaceptables. El exceso de peso fue mayor en el área urbana $(24,49 \%)$, que en la rural $(6,31$ $\%)$. Esta tendencia fue similar a la descrita en un estudio en México, donde el exceso de peso en el área urbana fue de $23,02 \%$ y, en la rural, de $11,71 \%$ (28), y en China, con 27,8 y $20,9 \%$, respectivamente (29). Estos resultados podrían explicarse por un mayor sedentarismo (cuadro 3, figura 1) y, probablemente, por las diferencias en los patrones de dietas en el área urbana.

Aunque en el presente estudio no se evaluó el nivel socioeconómico, otra hipótesis que explicaría este hallazgo sería que en Colombia las condiciones socioeconómicas de la población rural son menos favorables (17), y esta presenta una menor prevalencia de sobrepeso y obesidad que la población urbana (9).

En los niños con exceso de peso, los valores de triglicéridos, la prevalencia del sedentarismo y la hipertensión arterial sistémica fueron mayores que en los niños sin exceso de peso, lo que coincide con lo reportado en Estados Unidos (21). La diferencia en la prevalencia de hipertensión arterial sistémica entre los niños con exceso de peso y sin este fue casi del doble en este estudio, a diferencia de lo encontrado en un metaanálisis sobre prevalencia de hipertensión arterial en niños y adolescentes africanos, en el cual dicho aumento fue de casi seis veces (30). Este hallazgo puede deberse a la diferencia de edades en las poblaciones, así como al hecho de que África tiene una de las prevalencias de hipertensión más elevadas del mundo, probablemente asociada con factores genéticos (31). Estos resultados llaman a la adopción de estrategias de control del exceso de peso como medida de salud pública para la disminución de otros factores de riesgo (7).

La prevalencia del consumo de una dieta no saludable fue elevada, similar a lo observado en niños de Estados Unidos (27), aunque el uso de diferentes instrumentos de evaluación limita la comparación. Es probable que las diferencias en algunos factores ambientales, culturales y de comportamiento en las zonas rural y urbana estén relacionadas con los patrones de alimentación de la población en el presente estudio.

Una hipótesis que podría explicar la mayor prevalencia de sedentarismo en el área urbana, podría aludir a una menor disponibilidad de tiempo y de áreas para practicar actividad física, así como más tiempo de exposición a pantallas y teléfonos móviles, como se ha observado a nivel internacional (32), lo cual contribuye al exceso de peso y al aumento del riesgo de enfermedad cardiovascular en la edad adulta.

Otra hipótesis plantea que la diferencia se debe a que los niños en las áreas rurales se desplazan a sus escuelas caminando o en bicicleta. La mayor prevalencia de sedentarismo entre las niñas, tanto en el área rural como en la urbana, podría deberse a factores socioculturales, como la influencia de pares o la práctica de la actividad física por parte de sus madres, y a factores ambientales como la influencia de los medios de comunicación (33). 
Entre los niños sedentarios hubo una mayor prevalencia de hipertensión arterial sistémica y de exceso de peso, lo cual evidencia la importancia de la promoción de estilos de vida activos como estrategia de control de la aparición de factores de riesgo cardiovascular, siendo las estrategias más efectivas en los colegios aquellas que integran diversos componentes, como las clases de educación física, las pausas activas entre clases, las sesiones de actividades antes y después del colegio, y un desplazamiento activo entre el hogar y la escuela, y viceversa (34).

La prevalencia del tabaquismo pasivo, que constituye el mayor factor de riesgo cardiovascular modificable (35), fue alta en la población de estudio. Se estima que el $40 \%$ de los niños a nivel mundial están expuestos al humo de tabaco (36). En algunos estudios, se ha encontrado que los niños expuestos al tabaquismo pasivo tienen altos niveles de marcadores de reacción inflamatoria, bajos niveles de HDL, vitamina $\mathrm{C}$ y antioxidantes, y un elevado riesgo de enfermedad coronaria, de hasta 30 \% (37). La American Heart Association sugiere que los profesionales de atención primaria en salud deberían promover el abandono del tabaquismo, la reducción del tabaquismo pasivo y la prevención de su inicio (21). Es importante continuar promoviendo e implementando políticas antitabaco y enseñar a los niños la importancia de reclamar espacios libres de humo, empezando por sus hogares.

Los niños del área rural presentaron mayores niveles de colesterol total, de triglicéridos y de glucemia en ayunas, que los niños del área urbana, sin que la diferencia fuera estadísticamente significativa al ajustar por edad y sexo, lo cual coincide con lo descrito en estudios de China (38) y Ecuador (10). Aunque en el estudio no se hizo una encuesta de frecuencia de consumo de alimentos, los hallazgos podrían explicarse por una mayor ingestión de fritos y carbohidratos en el área rural, cuyo consumo en exceso provoca su transformación en triglicéridos que se depositan en el tejido adiposo. La ENSIN 2010 encontró que el $95,2 \%$ de los colombianos consumía fritos (32\% diariamente), el 98,6\% consumía azúcar, panela o miel (94,6\% diariamente), y el $98,3 \%$, tubérculos y plátano (66 \% diariamente), y que en todos los casos el consumo era mayor en el área rural. Además, aunque el consumo de alimentos de paquete, gaseosas, golosinas y dulces, era mayor en el área urbana, el consumo de fritos, azúcar, panela, miel, tubérculos y plátano, fuentes de almidón, lo era en el área rural (9). Estos hallazgos implican que las estrategias de salud pública en las áreas rurales deben ser diferentes a las del área urbana, y deben priorizarse los estilos de alimentación saludable.

Aunque la prevalencia de hipertensión arterial fue mayor en el área urbana que en la rural $(11,28$ Vs. $6,31 \%$ ) (cuadro 3), esta diferencia no fue significativa al ajustarla por edad y sexo (figura 1), datos estos similares a los reportados en México, en donde se encontró una prevalencia de 9,9\% en el área urbana y de $4,8 \%$ en la rural. Estos hallazgos pueden explicarse por una mayor prevalencia del exceso de peso y el sedentarismo en la población urbana, por lo que las estrategias de control del peso por medio de una alimentación saludable y una adecuada actividad física son una herramienta efectiva (27).

Algunas limitaciones del estudio residen en que la población de estudio no correspondió a una muestra aleatorizada de los niños en las poblaciones estudiadas en Colombia, lo cual conlleva un sesgo de selección e impide generalizar los datos a todo el país. Además, es posible que existieran factores distribuidos desigualmente entre las muestras $y$, por ende, responsables de las magnitudes de los resultados; aunque se ajustó por edad y sexo, es factible que haya habido una confusión residual debido a variables no medidas.

Dadas las diferencias en las definiciones utilizadas en este estudio y aquellas de la American Heart Association (cuadro 1), hubo una tendencia a sobreestimar la prevalencia del sedentarismo en el estudio $y$, en el caso de la dieta no saludable, la tendencia fue a subestimarla. El nivel socioeconómico, la raza y la etnia no se consideraron en el presente estudio, por lo que su relación con la presencia de factores de riesgo cardiovascular no se estimó. La comparación entre los colegios públicos del área rural y los colegios privados del área urbana podría haber agrandado las diferencias entre las poblaciones, dado que las condiciones socioeconómicas suelen ser menos favorables en el área rural (17). En futuros estudios el muestreo tendría que ser aleatorio, y deberían evaluarse el nivel socioeconómico y la frecuencia alimentaria, lo que permitiría la generalización y la explicación de algunos de los presentes hallazgos.

En conclusión, en este estudio los niños presentaron una elevada prevalencia de factores de 
riesgo cardiovascular, principalmente en el área urbana, donde la prevalencia de sedentarismo, sobrepeso y obesidad fue mayor que en el área rural, lo que sugiere una posible relación con patrones culturales y ambientales. El exceso de peso fue un factor común para otros factores de riesgo cardiovascular, como el sedentarismo y la hipertensión arterial, por lo que su prevención y su control deben traducirse en medidas de intervención en salud pública.

Los resultados del estudio, a pesar de sus limitaciones, respaldan la necesidad de diseñar políticas y programas de promoción de la salud cardiovascular desde la niñez, que tengan en cuenta las diferencias culturales, ambientales y de sexo en áreas rurales y urbanas, y apunten a reducir el desarrollo de factores de riesgo cardiovascular y la carga de la enfermedad en la adultez.

\section{Agradecimientos}

Agradecemosalos niños, lospadresy las autoridades escolares, por su generosa participación.

\section{Conflicto de intereses}

Los autores declaran no tener conflictos de intereses relacionados con este artículo.

\section{Financiación}

Este trabajo fue parcialmente financiado por una subvención de Colciencias al programa CARDIECOL (696-2014), por la Fundación Cardio-Infantil Instituto de Cardiología y por la Fundación Antonio Puerto.

\section{Referencias}

1. Adeyi O, Smith O, Robles S. Public policy and the challenge of chronic noncommunicable diseases. Washington, D.C.: The International Bank for Reconstruction and Development/ The World Bank; 2007. p. 1-218.

2. World Health Organization. Global strategy on diet, physical activity and health: A framework to monitor and evaluate implementation.Geneva: World Health Organiza tion; 2006. Fecha de consulta: 10 de abril de 2018. Disponible en: http://www.who.int/dietphysicalactivity/lndi cators English.pdf

3. Nicklas TA, Morales M, Yang SJ, Zakeri I, Berenson GS. Tracking of overweight status from childhood to young adulthood: The Bogalusa Heart Study. Eur J Clin Nutr. 2006; 60:48-57. https://doi. org/10.1038/sj.ejcn.1602266

4. Berenson GS, Srinivasan SR, Bao W, Newman WP, Tracy RE, Wattigney W. Association between multiple cardiovascular risk factors and atherosclerosis in children and young adults: The Bogalusa Heart Study. N Engl J Med. 1998;338:1650-6. https://doi.org/10.1056/NEJM199806043 382302
5. Abegunde DO, Mathers CD, Adam T, Ortegon M, Strong $\mathrm{K}$. The burden and costs of chronic diseases in low-income and middle-income countries. Lancet. 2015;370:1929-38. https://doi.org/10.1016/S0140-6736(07)61696-1

6. Raymond SU, Leeder S, Greenberg HM. Obesity and cardiovascular disease in developing countries: A growing problem and an economic threat. Curr Opin Clin Nutr Metab Care. 2006;9:111-6. https://doi.org/10.1097/01.mco.000021 4568.52192 .91

7. Lloyd, L J. Langley-evans, SC. Mcmullen S. Childhood obesity and risk of the adult metabolic syndrome: A systematic review. Int J Obes. 2012;36:1-11. https://doi.org/ 10.1038/ijo.2011.186

8. Lim SS, Allen K, Dandona L, Forouzanfar MH, Fullman $\mathbf{N}$, Goldberg $\mathbf{E}$, et al. Measuring the health-related Sustainable Development Goals in 188 countries: A baseline analysis from the Global Burden of Disease Study 2015. Lancet.2016;388:1813-50.https://doi.org/10.1016/S0140-67 36(16)31467-2

9. Ministerio de Salud y Protección Social, Instituto Colombiano de Bienestar Familiar. Encuesta Nacional de la Situación Nutricional en Colombia 2010. Bogotá: ICBF; 2010. p. 512.

10. Ochoa A, Andrade S, Huynh T, Verstraeten R, Lachat C, Rojas R, et al. Prevalence and socioeconomic differences of risk factors of cardiovascular disease in Ecuadorian adolescents. Pediatr Obes. 2012;7:274-83. https://doi.org/ 10.11 11/j.2047-6310.2012.00061.x

11. Cândido AP, Benedetto R, Castro AP, Carmo JS, Nicolato $\mathbf{R}$, Nascimento-Neto $\mathbf{R}$, et al. Cardiovascular risk factors in children and adolescents living in an urban area of Southeast of Brazil: Ouro Preto Study. Eur J Pediatr. 2009;168:1373-82. https://doi.org/10.1007/s00431-009-0940-1

12. Casanueva V, Cid X, Chiang MT, Román R, Milos C, Reyes $\mathbf{M}$,et al. Serum lipid levels in children and teenagers from Concepción, Chile. Rev Med Chil. 1996;124:1453-61.

13. Juárez JG, Cardoso G, Posadas R, Medina A, Yamamoto L, Posadas C. Blood pressure and associated cardiovascular risk factors in adolescents of México City. Arch Cardiol México. 2008;78:384-91.

14. Briceño G, Fernández $\mathbf{M}$, Céspedes J. Prevalencia elevada de factores de riesgo cardiovascular en una población pediátrica. Biomédica. 2015;35:219-26. https:// doi.org/10.7705/biomedica.v35i2.2314

15. Departamento Administrativo Nacional de Estadística. Distribución poblacional en el territorio y relaciones rrbanoregionales. Bogotá, D.C.: DANE; 2017. Fecha de consulta: 18 de julio de 2017. Disponible en: https://geoportal.dane. gov.co/atlasestadistico/pages/tome01/tm01 itm15.html

16. The World Bank Group. Rural population. Washington, D.C.: The World Bank Group; 2017. Fecha de consulta: 21 de septiembre de 2017. Disponible en: https://data.world bank.org/indicator/SP.RUR.TOTL

17. Pineda B. Desarrollo humano y desigualdades en salud en la población rural en Colombia. Universitas Odontológica. 2012;31:97-102. 
18. Xu S, Ming J, Yang C, Gao B, Wan Y, Xing Y,et al. Ur ban, semi-urban and rural difference in the prevalence of metabolic syndrome in Shaanxi province, northwestern China: A population-based survey. BMC Public Health. 2014;14:104. https://doi.org/10.1186/1471-2458-14-104

19. UNICEF. Children in an Urban World: The State of the World's Children 2012. New York,: UNICEF; 2012. p. 107. Fecha de consulta: 10 de abril de 2018. Disponible en: http://www.unicef.org/sowc2012/pdfs/SOWC 2012-Main Re port_EN_13Mar2012.pdf

20. Expert Panel on Integrated Guidelines for Cardiovascular Health and Risk Reduction in Children and Adolescents; National Heart, Lung, and Blood Institute. Expert panel on integrated guidelines for cardiovascular health and risk reduction in children and adolescents: Summary report. Pediatrics. 2011;128(Supl.5):S213-56. https://doi. org/10.1542/peds.2009-2107C

21. Steinberger J, Daniels SR, Hagberg N, Isasi CR, Kelly AS, Lloyd-Jones D, et al. Cardiovascular health promotion in children: Challenges and opportunities for 2020 and beyond. A scientific statement from the American Heart Association. Circulation. 2016;134:e236-55. https://doi.org/ 10.1161/CIR.0000000000000441

22. Centers for Disease Control and Prevention. Global school-based student health survey (GSHS). Questionnaire. 2013. Fecha de consulta: 12 de noviembre de 2017. Disponible en: https://www.cdc.gov/gshs/questionnaire/index.htm

23. National High Blood Pressure Education Program Working Group on High Blood Pressure in Children and Adolescents. The fourth report on the diagnosis, evaluation, and treatment of high blood pressure in children and adolescents. Pediatrics. 2004;114:555-76.

24. World Health Organization. Training Course on child growth assessment, WHO Child Growth Standards. Gene va: WHO; 2008.

25. Ministerio de Salud y Protección Social, Instituto Nacional de Salud, Instituto Colombiano de Bienestar Familiar. Encuesta Nacional de la Situación Nutricional. ENSIN 2015 Bogotá D.C.: Ministerio Nacional de Salud; 2017.p. 1-62. Fecha de consulta: 23 de mayo de 2018. Disponible en: https://www.minsalud.gov.co/Paginas/Gobie rno-presenta-Encuesta-Nacional-de-Situación-Nutricionalde-Colombia-ENSIN-2015.aspx

26. Ogden CL, Carroll MD, Kit BK, Flegal K. Prevalence of childhood and adult obesity in the United States, 20112012. JAMA. 2014;311:806-14. https://doi.org/10.1001/ja ma.2014.732

27. Ning H, Labarthe DR, Shay CM, Daniels SR, Hou L, Van Horn L, et al. Status of cardiovascular health in US children up to 11 years of age: The National Health and Nutrition Examination Surveys 2003-2010.Circ Cardiovasc Qual Outcomes. 2015;8:164-71. https://doi.org/10.1161/CIRCO UTCOMES.114.001274
28. Yamamoto-Kimura L, Posadas-Romero C, PosadasSánchez R, Zamora-González J, Cardoso-Saldaña G, Méndez-Ramírez I. Prevalence and interrelations of cardiovascular risk factors in urban and rural Mexican adolescents. J Adolesc Health. 2006;38:591-8. https://doi. org/10.1016/j.jadohealth. 2005.04.004

29. Yang Y, Hu XM, Chen TJ, Bai MJ. Rural-urban differences of dietary patterns, overweight, and bone mineral status in Chinese students. Nutrients. 2016;8. https://doi.org/10.33 90/nu8090537

30. Noubiap JJ, Essouma M, Bigna JJ, Jingi AM, Aminde LN, Nansseu JR. Prevalence of elevated blood pressure in children and adolescents in Africa: A systematic review and meta-analysis. Lancet Public Health. 2017;2:e375-e386. https://doi.org/10.1016/S2468-2667(17)30123-8

31. Yako YY, Balti EV, Matsha TE, Dzudie A, Kruger D, Sobngwi E, et al. Genetic factors contributing to hypertension in African-based populations: A systematic review and meta-analysis. J Clin Hypertens (Greenwich). 2018;20:485-95. https://doi.org/10.1111/jch.13225

32. Wolf AM, Gortmaker SL, Cheung L, Gray HM, Herzog DB, Colditz GA. Activity, inactivity, and obesity: Racial, ethnic, and age differences among schoolgirls. Am J Public Health. 1993;83:1625-7.

33. Sallis JF, Prochaska J, Taylor W. A review of correlates of physical activity of children and adolescents. Med Sci Sport Exerc. 2000;32:963-75

34. Physical Activity Guidelines for Americans Midcourse Report Subcommittee of the President's Council on Fitness, Sports and Nutrition. Physical Activity Guidelines for Americans Midcourse Report Strategies to Increase Physical Activity Among Youth. U.S. Washington, D.C.: Department of Health \& Human Services;2012.

35. Wang D, Juonala M, Viikari JS, Wu F, Hutri-Kähönen $\mathbf{N}$, Raitakari OT, et al. Exposure to parental smoking in childhood is associated with high c-reactive protein in adulthood: The cardiovascular risk in young Finns study. J Atheroscler Thromb. 2017;24:1231-41. https://doi.org/ 10.5551 /jat.40568

36. Oberg M, Jaakkola MS, Woodward A, Peruga A, PrüssUstün A. Worldwide burden of disease from exposure to second-hand smoke: A retrospective analysis of data from 192 countries. Lancet. 2011;377:139-46. https://doi. org/10.1016/S0140-6736(10)61388-8

37. Barnoya J, Glantz S. Cardiovascular effects of second hand smoke nearly as large as smoking. Circulation. 2005;111:2684-98. https://doi.org/10.1161/CIRCULATIONA HA.104.492215

38. McCarthy K, Cai LB, Xu FR, Wang PG, Xue HL, Ye YL,et al. Urban-rural differences in cardiovascular disease risk factors: A cross-sectional study of schoolchildren in Wuhan, China. PLoS One. 2015;10:e0137615. https://doi. org/10.1371/journal.pone.0137615 